\title{
Study and Search for Main Reason of Lung Cancers Based on Cherenkov Radiation in Environmental Radiation
}

\author{
Hiroshi Ito, Yusaku Emoto, Kento Fujihara, Hideyuki Kawai, Shota Kimura, Satoshi Kodama and Takahiro \\ Mizuno
}

\begin{abstract}
The number of lung-cancer-related death is highest among all cancers in the world, and it is increasing in Japan where population aging in progressing. The main reason for the lung cancer of non-smokers is regarded to be environmental pollution or exposure of the lung to radon in the nature. The risk of lung cancer was estimated to increase by 8 to $13 \%$ per every $100 \mathrm{~Bq} \mathrm{~m}^{-3}$ concentration of radon in the air. We observed beta rays with maximum energy of $3.27 \mathrm{MeV}$ emitted from ${ }^{214} \mathrm{Bi}$ as one of the progenies based on a detection of Cherenkov radiation. The surface radioactivity concentration of ${ }^{214} \mathrm{Bi}$ on the sample was measured; the relation between the concentration and exposure time for the sample at the room air is researched. The behavior of the radon progenies in the air is discussed by a research for the progenies attaching on the sample after the radon decay. The inhalation of the radon progenies is not clear. Thus, to understand the behavior of progenies in the air make to clear the causal relation between the radon concentration and lung cancers.
\end{abstract}

Index Terms-Radon Progenies, Bismuth-214, Cherenkov Radiation

\section{INTRODUCTION}

$\mathbf{T}$ HE number of lung-cancer-related death is highest among all cancers in the world, and it is increasing in Japan where population aging in progressing [1]. Although the primal cause of lung cancer is considered to be smoking, non-smokers also suffer. The main reason for the lung cancer of non-smokers is regarded to be environmental pollution or exposure of the lung to radon in the nature [3], [4]. The risk of lung cancer was estimated to increase by 8 to $13 \%$ per every $100 \mathrm{~Bq} \mathrm{~m}^{-3}$ concentration in the air, thus showing the strong possibility of decease causing due to inhalation. This result was extracted from the relation between the patient rate and the radon concentration in the North American, European, and Chinese [5]. Thus, the inhalation of radon is a strong candidate for the occurring of the lung cancer in the nature.

The average of annual radioactive dose is estimated as $1.15 \mathrm{mSv}$ from the ${ }^{222} \mathrm{Rn}$ inhalation at the mean concentration of $45 \mathrm{~Bq} \mathrm{~m}^{-3}$ in the air [6]. This value depends on the equilibrium factor after the radon is inhaled [7], which in assumed to be 0.4 , for radon progenies of ${ }^{218} \mathrm{Po},{ }^{214} \mathrm{~Pb}$, ${ }^{214} \mathrm{Bi}$, and ${ }^{214} \mathrm{Po}$ in radon decaying. However, it is not clear in the papers [6], [8] how the inhalation of theses progenies was treated if they are in the air and inhaled. The inhalation

Manuscript received 1 June, 2017.

H. Ito is with the Graduate of School of Science, Chiba University, Chiba, 268-8522, Japan (e-mails: hiroshi@ hepburn.s.chiba-u.ac.jp). radiative dose can be estimated more in precise if the behavior and concentration of these progenies in the air become to known.

The radionuclides of ${ }^{214} \mathrm{Bi}$, which emit beta rays (max. kinetic energy of $3.27 \mathrm{MeV}$ ) are focused in this study. The detection of beta rays in environment has an advantage because a concentration of radioactivity of the radon progenies can be measure mainly, i.e., the behavior of the progenies can be discussed after radon decaying. In this present study, a gamma ray energy spectroscopy in the air and a surface contamination inspection for a sample based on scintillation detection were performed.

\section{EXPERIMENT}

The experimental setup is shown in Fig. 1. The sample (polyethlene) is set under the scintillating fibers after it was exposed in the room air with windless. The scintillating fiber (Kuraray, SCSF-78MJ, $\phi 0.2 \mathrm{~mm}$ ) is emitted photons when the charged particles deposit energies there. The fibers are assembled to a sheet with an effective area of $10 \times 10 \mathrm{~cm}^{2}$. The photons propagating by a total reflection in the fibers are observed by photomultiplier tubes (PMT): PMT1 and PMT2 (Hamamatsu, R9880U-210), which connect to both ends of the fibers. A Cherenkov detector using silica aerogel was set over the scintillating fibers. The aerogel (a refractive index of $1.041 \pm 0.001$, the size of $9.6 \times 9.6 \times 1 \mathrm{~cm}^{3}$ ) emits Cherenkov photons by a beta ray with kinetic energy over $1.31 \mathrm{MeV}$. The photons are lead to PMT3 or 4 (Hamamatsu, R125003 and H6528) by a reflector with aluminised Mylar and are observed. The black paper sets under the silica aerogel because the Cherenkov light originated from cosmic muons must not be reflect. The scintillation detector was set over the Cherenkov detector. It consists of a plastic scintillator (the size of $10 \times 10 \times 0.5 \mathrm{~cm}^{3}$ ), acrylic light guide, and PMT (Hamamatsu, H1161). To suppress events related to cosmic muon, the cosmic-ray-veto counter was set over aluminium plate with thick of $1 \mathrm{~cm}$. The veto counter consists of a plastic scintillator (the size of $10 \times 20 \times 0.5 \mathrm{~cm}^{3}$ ), acrylic light guides, and PMT6, 7 (Hamamtsu, H1161). The PMTs connected to both ends of the scintillator via the light guides.

Therefore, the beta rays originated from ${ }^{214} \mathrm{Bi}$ can be observed as events when the coincidence is satisfied followings: hit the scintillating fibers, emitted Cherenkov radiation, hit the scintillator detector, and not hit the veto counter. 


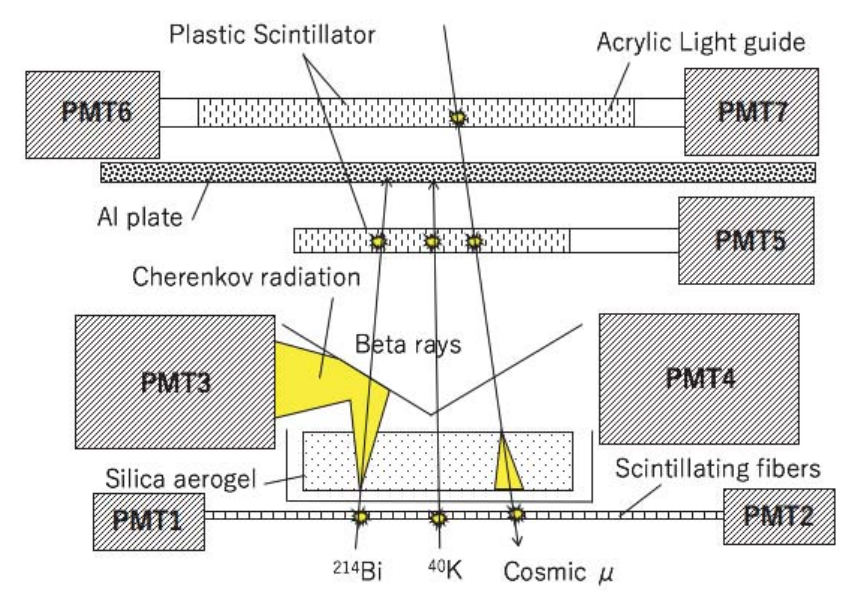

Fig. 1. A schematic of experimental setup.

\section{RESUlts AND Discussions}

The sample was exposed for $6 \mathrm{~h}$ in the room air with windless. As a result, the spectra of count rate with $10 \mathrm{~min}$ is shown in Fig. 2. The black dot is data. The red solid line is fitting exponential function. The green dashed line represents the background rate. The half-life time constant of the simple exponential function is $39.5 \pm 2.2 \mathrm{~min}$. The count rate related to ${ }^{214} \mathrm{Bi}$ becomes to reduce to un-observation amounts after late $4 \mathrm{~h} 40 \mathrm{~min}$.

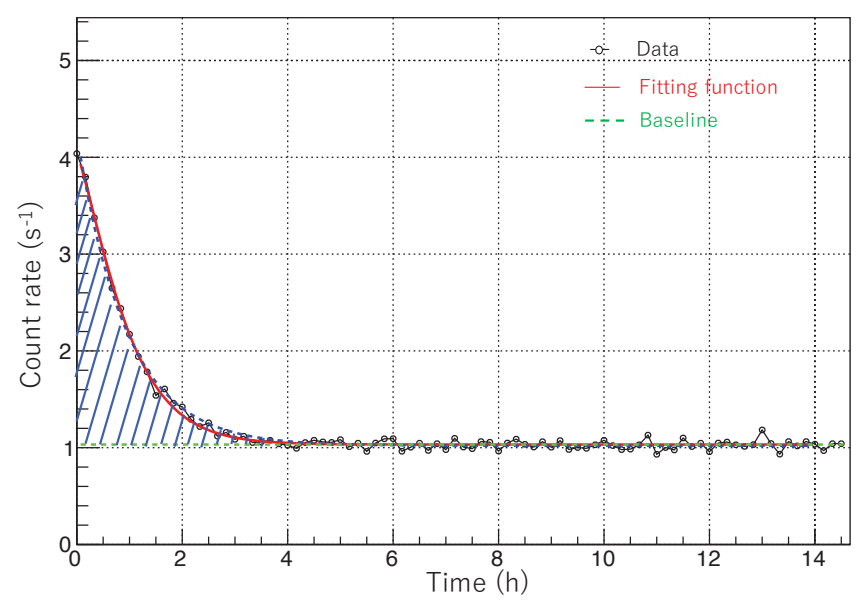

Fig. 2. The time spectrum of count rates for the polyethylene sample.

The integral blue shaded area responds to radioactivity concentration on the sample. This radioactivity is estimated by using corrected efficiency for ${ }^{214} \mathrm{Bi}$, where the efficiency is calculated from the experimental efficiency for ${ }^{90} \mathrm{Sr}$ and a ratio of efficiency for ${ }^{214} \mathrm{Bi}$ to that for ${ }^{90} \mathrm{Sr}$ by a Monte Carlo simulation, GEANT4. As a result, this radioactivity (4 h 40 min exposes) is $18.0 \pm 0.5 \mathrm{~Bq} / \mathrm{m}^{2}$.

The relation between the radioactivity concentration and exposure time is shown in Fig. 3. The black dot is data and the red solid line is fitting function: $(17.9 \pm 0.5)\{1-\exp (-(t /(1.68 \pm 0.06)))\}$. This result sug- gests that ${ }^{214} \mathrm{Bi}$ of $17.9 \pm 0.5 \mathrm{~Bq} / \mathrm{m}^{2}$ attaches on our surroundings and the concentration becomes to be saturated just after the radon decay in the air. Furthermore, it also suggests the radon progenies $\left({ }^{218} \mathrm{Po},{ }^{214} \mathrm{~Pb},{ }^{214} \mathrm{Bi}, 214 \mathrm{Po}\right.$, etc. $)$ do not fall down to ground, and some components behavior in the air. Therefore, the potential by inhaling the progenies should be considered for natural exposure dose.

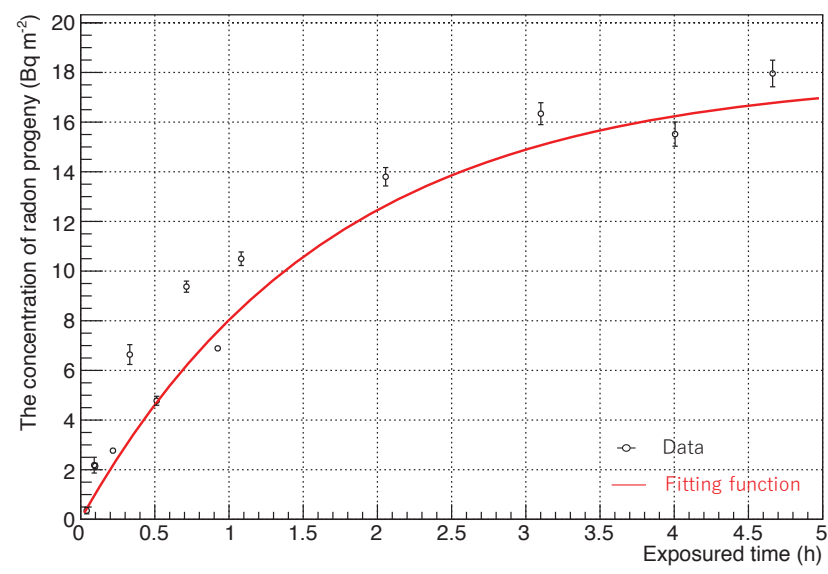

Fig. 3. The relation between the radioactivity concentration and exposure time.

\section{SUMMARY}

The radon progenies in the air is focused by the recent study. The behavior of radon progenies is discussed indirectly by measuring the radioactivity concentration of ${ }^{214} \mathrm{Bi}$ on the sample based on Cherenkov detection. The results suggest the potential of inhaling radon progenies. In future, the radioactivity of radon progenies in air will be measured directly.

\section{REFERENCES}

[1] Foundation for Promotion of Cancer Research, 2015. Cancer statistics in Japan 2015.

[2] Funatogawa I., Funatogawa T., Yano E., 2013. Bull World Health Organ. 91 (2013) 332-340.

[3] Subramanianand J., Govindan R., 2007. Lung Cancer in Never Smokers: A Review. J. Clin. Oncol., 25 (2007) 561-570.

[4] Samet J. M., Avila-Tang E., Boffetta P., Hannan L. M., Olivo-Marston S., Thun M. J., Rudin C. M., 2009. Lung Cancer in Never Smokers: Clinical Epidemiology and Environmental Risk Factors. Clin. Cancer. Res. 15 (18) (2009) 5626-5645.

[5] Choi H., Mazzone P., 2014. Radon and lung cancer: Assessing and mitigating the risk. Clev. Cli. J. 81 (9) (2014) 567-575.

[6] UNSCEAR 2008 report vol. 1. Source effects and risks of ionizing radiation.

[7] Martinez J. E., Juste B., Ortiz J., Martorell S., Verdu G., 2017. Air Radon equilibrium factor measurement in a Waste Water Pre-Treatment Plant. Rad. Phys. Chem. 2017.

<https://doi.org/10.1016/j.radphyschem.2017.03.011>.

[8] ICRP publication 71. Age-dependent Doses to Members of the Public from Intake of Radionuclides: Part 4 Inhalation Dose Coefficients. A report of a Task Group of Committee 2 of the International Commission on Radiological Protection. Adopted By The Commission In September 1995. 